\title{
REGRAS DE PUBLICAÇÃO EM REVISTAS BIOMÉDICAS: SUA CONFORMIDADE COM A "PROVA DOS NOVE"
}

\section{RULES OF PUBLICATION IN BIOMEDICAL JOURNALS: THEIR CONFORMITY WITH “LITMUS TEST”}

\author{
Julio César Lima Sampaio ${ }^{\text {, Magnaura dos Santos }}{ }^{I I}$, Suely Oliveira Moraes Marquez ${ }^{I I I}$ \\ Vanusa Jardim Borges ${ }^{I V}$
}

Recebido em: 11-02-2019

Aceito em: 08-04-2019

\begin{abstract}
Resumo
Descreve o resultado de pesquisa sobre se as regras de publicação de periódicos biomédicos são fonte segura para orientar autores de artigos no ajuste de itens de manuscritos a serem submetidos. Trata-se de estudo quantitativo cujos objetivos foram identificar os elementos essenciais na composição das palavras-chave, citações e referências e avaliar a conformidade das regras de publicação das revistas comparando-as com a estrutura dos artigos publicados nos fascículos mais recentes. Procedimentos denominados pelo autor como sendo a "Prova dos nove". Foram incluídas na pesquisa revistas de Dermatologia que seguem as recomendações do International Committee of Medical Journal Editors e que disponibilizam suas regras em português, inglês, francês ou espanhol. A análise das 24 revistas incluídas no estudo mostrou que as regras de publicação não são totalmente observadas pelas revistas de dermatologia que informam seguir as recomendações da norma de Vancouver, sendo necessário, para uma maior segurança dos autores, consultar os artigos mais recentes, a fim de utilizá-los como orientação adicional na formatação de seus manuscritos.
\end{abstract}

Palavras-chave: Regras de publicação. Normas para autores. Norma de Vancouver

\begin{abstract}
The present study describes the search result on whether biomedical journals publication rules are a safe source to guide article authors in adjusting manuscript items to be submitted. This paper is a quantitative study whose objectives were to identify the essential elements in the composition of the keywords, citations, and references to evaluate journals and their publication rules, regarding its conformity also comparing them with the most recent published essays and their structure. Procedures named by the author as the "Litmus test". Included in the research were Dermatology journals that follow the recommendations of the International Committee of Medical Journal Editors and make their rules available in portuguese, english, french or spanish. The 24 journals included in this study and their analysis reflects that the rules of publication have not been fully observed by the dermatology journals that report attending the Vancouver standards. Concerning the interest of authors safety, it is crucial to consult the most recent articles to use them as additional guidance in the formatting of their manuscripts.
\end{abstract}

Keywords: Publishing rules. Rules for authors. Vancouver Standard

\footnotetext{
' Fundação de Dermatologia Tropical e Venereologia Alfredo da Matta - e-mail: julioclsampaio@gmail.com

" Bibliotecária- UFAM - email: magnaurasantos@gmail.com

III Mestre em Sociedade e Cultura da Amazônia - Professora da Universidade Federal do Amazonas - e-mail: biblioteca@fuam.am.gov.br

IV Mestre em Sociedade e Cultura da Amazônia Professora da Universidade Federal do Amazonas - e-mail: biblioteca@fuam.am.gov.br
} 


\section{INTRODUÇÃO}

A comunicação escrita é considerada como memória da humanidade e deve ajustar-se aos diferentes tipos de leitores a fim de atingir o fim almejado. O insumo básico que proporciona o desenvolvimento científico e tecnológico de uma nação é a informação que ela produz como resultado de pesquisas diversas divulgadas à comunidade em periódicos, através da utilização de procedimentos adotados pelo sistema de comunicação científica (KURAMOTO, 2006; SILVA, 1993).

Os procedimentos adotados pelas revistas para publicação de artigos científicos são comumente denominados de regras para autores, instruções para autores, normas de publicação entre outros. Nestes, encontram-se orientações de caráter intrínseco (conteúdo) e extrínseco (forma). Entre as recomendações intrínsecas estão as que definem o papel de cada um dos autores, questões sobre ética, metodologia, resultados e considerações finais. Em relação à forma do manuscrito verifica-se, por exemplo, a quantidade de palavras no título, no resumo e nas palavras-chave, bem como o formato das citações e das referências (LEITE, 2009; VOLPATO, 2015).

A publicação de artigos é o desejo de todo pesquisador. Contudo, o momento de compartilhar e difundir as pesquisas no meio acadêmico requer algumas decisões, tais como, onde publicar (anais de eventos ou periódicos científicos) e em qual evento ou periódico publicar. No caso dos periódicos, comumente, a análise da revista deverá ser feita com base nos seguintes fatores: frequência da publicação; audiência; objeto e escopo; fator de impacto entre outros. Além de muita leitura sobre o assunto e analisar questões éticas envolvidas na pesquisa, o pesquisador terá ainda que estudar a formatação (regras de publicação) e a documentação necessária exigida pelo periódico (LEITE, 2009; SANTOS, 2014; SILVEIRA, 2009; VOLPATO, 2015).

Em relação às regras de publicação, espera-se que sejam confiáveis e tenham linguagem clara e atualizada para que os autores possam tê-las como guias de conteúdo e qualidade de informações. É, obviamente necessária, a observação e o monitoramento dessas diretrizes pela própria revista, sob o risco de influenciar os autores a não investir tanto tempo e esforços na formatação (LEITE, 2009; SANTOS, 2014; SILVEIRA, 2009; VOLPATO, 2015).

A formatação de trabalhos acadêmicos, artigos de periódicos e outros, conta com várias normas para orientação. No Brasil, as mais conhecidas são as normas publicadas pela Associação Brasileira de Normas Técnicas (ABNT). Contudo, as publicações biomédicas contam com regra específica, comumente chamada de Norma de Vancouver (NV) ou Estilo Vancouver ${ }^{1}$, destinada a orientar autores da área biomédica na elaboração e formatação de manuscritos.

A NV é amplamente conhecida como o padrão de formatação das referências e citações de publicações biomédicas. O International Committee of Medical Journal Editors (ICMJE), responsável pela atualização e divulgação da norma, recomenda sua utilização para orientar as práticas éticas na condução e nos relatos de pesquisa e outros materiais publicados por periódicos biomédicos, além de auxiliar autores, editores e outros envolvidos na revisão por pares, a fim de propiciar que as publicações possam criar e distribuir informações precisas, claras, seguras e reprodutíveis (INTERNATIONAL COMMITTEE OF MEDICAL JOURNAL EDITORS, 2016, 2017a, 2017b, 2018).

A NV é um conjunto dividido em quatro capítulos. No primeiro capítulo encontram-se uma apresentação, o público alvo e um pequeno histórico da norma. O capítulo seguinte apresenta as funções e responsabilidades dos autores, colaboradores, revisores, editores e proprietários. No capítulo seguinte a NV apresenta os tipos de publicação e edições editoriais relacionadas às publicações em periódicos biomédicos. E, por fim, no quarto capítulo

\footnotetext{
${ }^{1}$ Recomendations for the Conduct, Reporting, Editing, and Publication of Scholarly Work in Medical Journals. Disponível em: http://www.icmje.org/recommendations
} 
encontram-se as orientações quanto à preparação e submissão de manuscritos (INTERNATIONAL COMMITTEE OF MEDICAL JOURNAL EDITORS, 2018).

O último capítulo da NV divide-se em dois subitens: Subitem A. Preparação de Manuscritos para serem Submetidos aos Periódicos Médicos e Subitem B - Submetendo Manuscritos aos Periódicos Médicos. O subitem A é mais completo e contém além dos princípios gerais, orientações sobre os critérios para construção do título de um artigo; a estrutura e características do resumo; introdução; metodologia; estatísticas; resultados; discussão; referências e outros (INTERNATIONAL COMMITTEE OF MEDICAL JOURNAL EDITORS, 2018).

Para efeito de análise, consideraremos neste artigo somente as orientações no item Referências subdivididas em dois grupos: considerações gerais (item i) e estilo e formato (item ii). É, portanto no item Referências que estão as orientações básicas para redação das referências bibliográficas assim como das citações (INTERNATIONAL COMMITTEE OF MEDICAL JOURNAL EDITORS, 2018).

No corpo do texto das orientações sobre o estilo e o formato das referências da NV há dois $l i n k s^{2}$ para acesso à lista de exemplos de referências mais comuns utilizadas em periódicos biomédicos e ao Citing Medicine ${ }^{3}$ que fornece detalhes da construção das referências no estilo de Vancouver (PATRIAS, 2007).

O ICMJE recomenda que o estilo das referências seja elaborado conforme o National Information Standards Organization (NISO) Z39.29-2005 (R2010) Bibliographic References adaptada da National Library of Medicine (NLM). Os detalhes do formato utilizado podem ser vistos na figura 1. Adicionalmente, a NV recomenda que as referências sejam numeradas na ordem em que elas aparecem no texto (INTERNATIONAL COMMITTEE OF MEDICAL JOURNAL EDITORS, 2016; PATRIAS, 2007).

Figura 1 - Exemplo do formato de referência de um artigo de revista, incluindo os sinais de pontuação utilizados

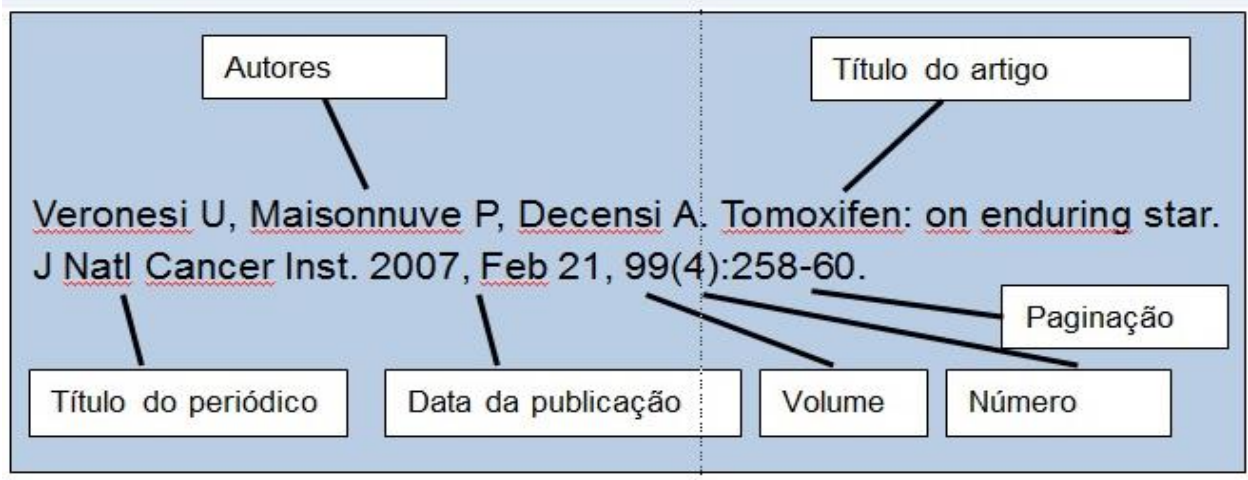

Fonte: Fonte: Adaptado de PATRIAS, 2007

$\mathrm{Na}$ abreviação dos títulos de periódicos biomédicos, a NV orienta os autores a consultarem a List of Journal Indexed for MEDLINE ${ }^{4}$. As normas brasileiras equivalentes à orientação quanto ao formato das citações e a abreviação de títulos de periódicos são respectivamente a NBR 10520:2002 e a NBR 6032:1989 (ASSOCIAÇÃO BRASILEIRA DE NORMAS TÉCNICAS, 1989, 2002; INTERNATIONAL COMMITTEE OF MEDICAL JOURNAL EDITORS, 2016; PATRIAS, 2007;).

\footnotetext{
2 Samples of Formatted References for Authors of Journal Articles. Disponível em: https://www.nlm.nih.gov/bsd/uniform_requirements.html.

${ }^{3}$ Citing Medicine. Disponível em: https://www.ncbi.nlm.nih.gov/books/NBK7256/.

${ }^{4}$ Journals Indexed from the Online Version. Disponível em: https://www.nlm.nih.gov/bsd/journals/online.html
} 
Apesar da NV ser apenas uma recomendação, a maioria das revistas da área biomédica a utiliza como padrão em suas publicações. No entanto, o próprio NCBI afirma que não se responsabiliza pela veracidade de tais afirmações (INTERNATIONAL COMMITTEE OF MEDICAL JOURNAL EDITORS, 2016; UNIVERSIDADE DO OESTE PAULISTA, 2011).

Os vocabulários controlados são um tipo de linguagens de indexação utilizados para tratamento temático da informação. Os vocabulários controlados, são portanto instrumento importante na recuperação da informação, pois são responsáveis pela definição das palavraschaves ou pontos de acesso (DIAS, 2013; SIQUEIRA, 2011).

$\mathrm{Na}$ área biomédica, há dois vocabulários controlados bastante conhecidos da comunidade e também pelos profissionais da informação da área. $\mathrm{O}$ primeiro é o Medical Subject Headings (MeSh) ${ }^{5}$ vocabulário controlado para a área médica elaborado e atualizado pela National Library of Medicine (NLM), usado para indexação, catalogação e busca de artigos biomédicos no Medical Literature Analysis and Retrieval System Online (MEDLINE) ${ }^{6}$. Já os Descritores em Ciências da Saúde (DeCS) ${ }^{7}$, são utilizados para indexação de documentos nas bases de dados da Biblioteca Virtual em Saúde (BVS), que incluem a Literatura LatinoAmericana e do Caribe em Ciências da Saúde (LILACS), MEDLINE, Rede Pan-Americana de Informação e Documentação em Engenharia Sanitária e Ciências do Ambiente (REPIDISCA), Banco de Dados em Enfermagem (BDENF) entre outras. Para definição das palavras-chave, a NLM sugere a adoção do MeSh como vocabulário controlado (DESCRITORES EM CIÊNCIAS DA SAÚDE, 2018; MEDICAL SUBJECT HEADINGS, 2019; NOVILO ORTIZ, 2010).

Ainda em relação às palavras-chave, no momento da confecção de manuscritos, dois outros itens de caráter extrínsecos devem estar claros nas orientações para os autores: a quantidade (máxima e mínima) e os sinais de pontuação utilizados.

As orientações quanto às citações são de caráter extrínseco (Tabela 1). A Norma de Vancouver orienta que as referências devem ser numeradas consecutivamente na ordem em que são mencionadas no texto. A identificação das referências no texto, tabelas e legendas (citações), deve ser feita com números arábicos entre parêntesis. No entanto, a Norma de Vancouver não deixa claro se a composição do número deverá ser sobrescrito (mais utilizado) ou na mesma linha do texto principal.

Tabela 1 - Relação de itens intrínsecos e extrínsecos nas palavras-chave, citação e referências em publicações biomédicas e respectivos campos essenciais.

\begin{tabular}{|c|c|c|}
\hline Item & Intrínsecos (conteúdo) & Extrínsecos (forma) \\
\hline Palavra-chave & $\begin{array}{l}\text { Definição (vocabulário } \\
\text { controlado) }\end{array}$ & $\begin{array}{l}\text { Quantidade (mínima e máxima) } \\
\bullet \quad \text { Pontuação utilizada }\end{array}$ \\
\hline Citação & - & $\begin{array}{l}\text { - Sistema utilizado } \\
\text { (numérico ou autor-data) } \\
\text { - Se numérico: } \\
\text { Sobrescritolmesma linha } \\
\text { - "solto", entre colchetes, } \\
\text { parênteses, aspa ou outro sinal } \\
\text { gráfico } \\
\text { - Antes ou após a pontuação }\end{array}$ \\
\hline Referências & - & $\begin{array}{ll}\text { - } & \text { Autoria } \\
\text { - } & \text { Título do documento }\end{array}$ \\
\hline
\end{tabular}

\footnotetext{
${ }^{5}$ Medical Subject Headings (MeSH). Disponível em: https://www.ncbi.nlm.nih.gov/mesh

${ }^{6}$ Base de Dados Medline. Disponível em: https://www.nlm.nih.gov/bsd/pmresources.html.

${ }^{7}$ Descritores em Ciências da Saúde. Disponível em: http://decs.bvs.br/
} 


\begin{tabular}{|l|l|l|l|}
\hline & & $\begin{array}{l}\bullet \\
\bullet\end{array}$ & Título da revista \\
& & $\bullet \quad$ Paginação e outros \\
\hline
\end{tabular}

\section{MATERIAL E MÉTODOS}

Trata-se de um estudo quantitativo cujo objetivo foi identificar os elementos essenciais em cada um dos itens analisados; avaliar a conformidade das regras das revistas para palavraschave, citações e referências na estrutura dos artigos mais recentes publicados pelas revistas de dermatologia que seguem as recomendações do ICMJE ou Norma de Vancouver.

Inicialmente, foram identificados os elementos considerados essenciais para cada um dos itens avaliados (Tabela 2). Em seguida, foram identificados os periódicos que seguem as recomendações de Vancouver de acordo com dados do ICMJE. Com o uso do comando de atalho $\mathrm{Ctr}+\mathrm{F}$ do Windows na tela do computador, utilizou-se parte da palavra-chave dermatologia (dermat) para identificar as revistas de dermatologia.

A busca retornou um universo de 32 revistas que inicialmente foram numeradas a fim de facilitar o tratamento das informações (Tabela 2). Contudo, apenas 24 revistas foram incluídas no estudo. Das 32 revistas inicialmente selecionadas, 03 (37\%) apresentaram regras em língua diferente do português, inglês, francês ou espanhol e o restante, 05 (63\%) não disponibilizaram acesso às regras. Uma destas revistas foi excluída por um motivo digno de nota: a revista orienta o autor a escrever em qualquer formato, pois o trabalho de adequação às normas da revista é de responsabilidade do corpo editorial.

Tabela 2 - Lista das revistas disponibilizadas pelo ICMJE

\begin{tabular}{|c|l|}
\hline Número & \multicolumn{1}{|c|}{ Título da Revista } \\
\hline 1 & Advances in cosmetics and dermatology (list date 1/5/16) \\
\hline 2 & Advances in dermatology and allergology (list date 3/27/15) \\
\hline 3 & American journal of clinical dermatology (list date 4/8/08) \\
\hline 4 & Annales de dermatologie et de venereologie \\
\hline 5 & Archives of dermatology \\
\hline 6 & Australasian journal of dermatology \\
\hline 7 & Chinese journal of dermatolog \\
\hline 8 & Clinical Medicine Insights: dermatology (list date 2/1/12) \\
\hline 9 & Clinical research in dermatology: open access (list date 5/7/14) \\
\hline 10 & Clinical, cosmetic and investigational dermatology (list date 9/4/14) \\
\hline 11 & Dermatologia kliniczna (list date 11/24/10) \\
\hline 12 & Dermatologica \\
\hline 13 & Dermatology and cosmetic (list date 8/13/12) \\
\hline 14 & Dermatology aspects (list date 9/29/15) \\
\hline 15 & Dermatology review (list date 9/5/13) \\
\hline 16 & European medical journal - dermatology (list date 7/30/14) \\
\hline & \\
\hline
\end{tabular}




\begin{tabular}{|l|l|}
\hline 17 & Expert review of dermatology (list date 12/13/11) \\
\hline 18 & Global dermatology (god) (list date 2/24/15) \\
\hline 19 & Images en dermatologie (list date 10/20/15) \\
\hline 20 & Indian journal of clinical and experimental dermatology (list date 3/3/16) \\
\hline 21 & Indian journal of dermatology \\
\hline 22 & Indian journal of dermatology, venereology and leprology \\
\hline 23 & International journal of research in dermatology (list date 3/11/16) \\
\hline 24 & Investigative dermatology and venereology research (list date 5/25/16) \\
\hline 25 & Journal of clinical and investigative dermatology (list date 6/4/15) \\
\hline 27 & Journal of dermatologic research and therapy (list date 3/2/16) \\
\hline 28 & $\begin{array}{l}\text { Journal of the american academy of dermatology (list date 7/10/06) } \\
3 / 29 / 12 \text { ) }\end{array}$ \\
\hline 29 & Our dermatology online (list date 6/9/11) \\
\hline 30 & Research and reports in transdermal drug delivery (list date 9/16/14) \\
\hline 31 & The journal of the philippine dermatological society (list date 1/20/11) \\
\hline 32 & World journal of dermatology (list date 5/20/14) \\
\hline
\end{tabular}

Fonte: Adaptado de Journal Listing Request Form.

Disponível em: http://icmje.org/journals-following-the-icmje-recommendations/journal-listing-request-form/.

Foram incluídos na comparação somente os itens que estavam presentes na regra para os autores. Assim, como exemplo, se determinada revista apresentou em sua regra somente orientação para a quantidade de palavras-chave, a comparação foi realizada somente em relação a este item.

Os artigos utilizados para a "Prova dos nove" neste trabalho que não apresentaram nenhuma referência de livros ou outro tipo de monografia foram identificados com a sigla NAV (Não foi Possível Avaliar). Sendo assim, estes não foram contabilizados no somatório final para definição do percentual.

Após a seleção das revistas, a próxima etapa consistiu em acessar suas respectivas regras de publicações e numerá-las de acordo com o número previamente estabelecido na Tabela 2. Em seguida, foram obtidas cópias dos artigos mais recentes de cada uma das revistas para servirem de "Prova dos nove". O objetivo desta ação foi para comparar a conformidade da estrutura de cada um dos artigos com as determinações presentes nas regras de publicação de manuscritos para os autores.

As informações obtidas foram coletadas no período de julho a dezembro de 2016 e apresentadas em tabelas, organizadas e processadas segundo medidas da estatística descritiva obtidas pelo programa Microsoft Excel 2013. 


\section{RESULTADOS E DISCUSSÃO}

\subsection{PALAVRAS-CHAVE}

No item palavra-chave foram identificados e analisados a existência da necessidade de orientação para três elementos básicos: 1) quantidade de palavras possíveis (mínima e máxima), 2) formação (uso de vocabulário controlado ou não) e 3) sinal de pontuação utilizado para separar e finalizar as palavras-chave.

Os resultados mostraram que há deficiência na orientação com relação aos elementos essenciais referentes às palavras-chave, visto que nenhuma revista analisada forneceu orientação para todos os itens.

Das 24 revistas incluídas no estudo, apenas 13 (54\%) forneceram orientação satisfatória para ao menos um item (quantidade) em suas regras e 04 revistas (16\%) forneceram 2/3 das orientações básicas. Destas, apenas 03 revistas (75\%) possuíam orientação quanto à quantidade e formação e apenas 01 (25\%) apresentou regra para os itens 1 e 3 (quantidade e sinal de pontuação). $\mathrm{O}$ resultado mostra ainda que 07 revistas (30\%) não apresentaram orientação para nenhum dos itens considerados essenciais em relação às palavras-chave.

A análise comparativa mostrou que 21 revistas (87\%) mantiveram-se conformes com as suas regras; 03 revistas (13\%) apresentaram não conformidade em mais de um elemento (Revistas 4, 21 e 22). Neste caso, estas revistas apresentaram orientação para o item quantidade de palavras-chave, porém a análise da estrutura dos artigos analisados destas revistas não evidenciou a utilização desta orientação na prática.

Apenas 02 revistas (8\%) (Revistas 15 e 29), fornecerem em suas regras para autores, orientação quanto à fonte para escolha das palavras-chave. Nestes casos, observou-se que a fonte sugerida foi o MeSH.

\subsection{CITAÇÃO}

Em relação à citação, identificou-se a necessidade de quatro elementos básicos nas orientações para os autores: 1) sistema utilizado (numérico, autor-data ou outro); 2) se sobrescrito ou na mesma linha do texto; 3) numérico ("solto" ou entre parênteses, entre colchetes ou outro sinal); 4) Antes ou depois da pontuação (para ambos os sistemas).

A análise da existência ou não de orientação referente à citação revelou que apenas 03 $(12 \%)$ revistas apresentaram orientação completa.

Das 24 revistas, 06 (25\%) apresentaram 3/4 dos elementos; 09 (38\%) apresentaram 2/4 dos elementos; 05 (21\%) possuíam menos de 1/4 dos elementos básicos e 01 (4\%) não apresentou qualquer orientação (Revista 24).

$\mathrm{Na}$ análise da mostra de 24 revistas, observou-se outro fato digno de nota: apesar de todas as revistas incluídas na pesquisa figurarem na lista de periódicos que expressam seguir as recomendações do ICMJE, que recomenda a utilização do sistema numérico para citação, apenas 01 (4\%) afirmou não utilizar o sistema numérico (Revista 1), contrariando a sua existência na lista da ICMJE.

$\mathrm{Na}$ análise comparativa, percebeu-se que 20 (84\%) revistas mantiveram-se conforme as suas regras, incluindo a Revista 24 que, apesar de não fornecer nenhuma orientação quanto aos itens básicos, manteve-se conforme mesmo assim e 04 (16\%) apresentaram não conformidades em pelo menos um dos itens. 
Tabela 3 - Elementos essenciais e respectivas pontuações ortográficas analisadas para composição das palavras-chave e citações

\begin{tabular}{|c|c|}
\hline Item & \multicolumn{1}{c|}{ Informaçóes essenciais } \\
\hline \multirow{4}{*}{ Palavra-chave } & a) Quantidade: mínima e máximo; \\
& b) De onde elas são retiradas (Mesh, Decs, outro); \\
& c) Como elas são separadas (vírgula, ponto-e-vírgula, espaço, \\
& outro) \\
\hline \multirow{3}{*}{ Citação } & a) Numérica (arábico ou outro) ou Autor-Data; \\
& b) Sobrescrito ou na mesma linha do texto; \\
& c) Solto, entre parêntesis, colchetes, chaves ou outro sinal \\
& gráfico; \\
& d) Antes ou depois da pontuação \\
\hline
\end{tabular}

\subsection{REFERÊNCIAS}

As referências foram classificadas em nove grandes elementos básicos que, subdivididos, geraram dezenove subelementos: 1) autoria (livro, capítulo de livro e periódico); 2) uso ou não do et al; 3) data (livro, capítulo de livro e periódico); 4) título (livro, capítulo de livro e periódico); 5) editora (apenas para livro e capítulo de livro); 6) local de publicação (apenas para livro e capítulo de livro); 7) paginação (livro, capítulo de livro e periódico); 8) fascículo e número (apenas para artigos de periódicos) e 9) título do periódico.

A análise inicial identificou que $17(70 \%)$ revistas apresentaram orientação referente a todos os elementos e 07 (30\%) revistas possuíam deficiência em ao menos um elemento. Além deste resultado, 05 (72\%) não forneceram orientação quanto ao uso da expressão et. al.; 01 (14\%) não apresentou orientação para seis elementos básicos e 01 (14\%) não forneceu orientação para 13 elementos considerados essenciais.

A análise comparativa evidenciou que apenas 08 (33\%) mantiveram-se conformes com suas regras e $16(67 \%)$ apresentaram um ou mais itens em não conformidade.

Dos itens que apresentaram todos os elementos básicos, 08 (47\%) mantiveram-se conformes e $09(53 \%)$ apresentaram pelo menos uma não conformidade.

Apenas 04 revistas apresentaram 01 não conformidade cada uma (Revistas 12, 14, 15 e 16). A revista que apresentou mais problemas foi a de $n^{\circ} 24$ com mais de $60 \%$ dos itens não conformes, seguida da revista 18 com seis itens não conformes.

Observou-se que as não conformidades encontradas estavam distribuídas nos campos de autoria, data, título, editora, paginação, fascículo e título do periódico.

A existência de não conformidades nos três itens pesquisados sugere uma variedade de regras sendo utilizadas na prática, mas que não estão de acordo com as que estão sendo divulgadas para os autores.

\section{CONSIDERAÇÕES FINAIS}

As normas das revistas de dermatologia que orientam autores na formatação de manuscritos, ao menos em relação aos itens pesquisados, não são a única fonte segura para orientar autores na elaboração de manuscritos científicos, pois estas normas estão sendo descumpridas pelas próprias revistas.

Há uma variedade de regras contrariando a informação de que todas as revistas desta pesquisa utilizam a Norma de Vancouver. Na pesquisa, foi possível encontrar revista, por exemplo, que utiliza o sistema autor-data para as citações, quando no formato de Vancouver, o sistema utilizado é o numérico.

Seria fundamental a busca de um consenso entre as revistas biomédicas a fim de que seja utilizado um único formato, uma espécie de "Ctrl C e Ctrl V" da Norma de Vancouver, 
para que se evitem os vários diferentes modelos que prejudicam tanto os autores quanto a todo o corpo editorial.

Aos editores, sugere-se uma revisão de suas normas, disponibilizando a informação de suas atualizações. Sugere-se também que a ICMJE proceda a uma revisão da lista de periódicos que informam seguir as recomendações da ICJE e que sistematize uma verificação periódica das regras dessas revistas.

A sugestão do uso de vocabulário controlado (MeSH) foi orientada por um número pequeno de revistas, evidenciando a pouca importância dada a esta ferramenta. As revistas estão focando de forma exclusiva na quantidade mínima e máxima permitida para as palavras-chave.

A citação na Norma de Vancouver exige cuidados extras, visto que o formato numérico pode apresentar algumas variações: sobrescrito ou não; "solto", entre colchetes, entre parênteses ou outro sinal gráfico.

Enfim, até que se resolvam estas questões em relação às normas, e pelo fato de haver muitas não conformidades nas regras das revistas de dermatologia analisadas, sugere-se aos autores, que utilizem a estrutura dos artigos mais recentes das revistas que escolheram para publicar, para servirem como fonte adicional na orientação seus manuscritos.

\section{REFERÊNCIAS}

ASSOCIAÇÃO BRASILEIRA DE NORMAS TÉCNICAS. NBR 6032: abreviação de títulos de periódicos e publicações seriadas. Rio de Janeiro: ABNT, 1989.

ASSOCIAÇÃO BRASILEIRA DE NORMAS TÉCNICAS. NBR 10520: informação e documentação: citações em documentos: apresentação. Rio de Janeiro: ABNT, 2002.

DESCRITORES EM CIÊNCIAS DA SAÚDE: DeCS. 2018. ed. rev. e ampl. São Paulo: BIREME / OPAS / OMS, 2017. Disponível em: http://decs.bvsalud.org. Acesso em: 10 jan. 2019.

DIAS, Eduardo Wense; NAVES, Madalena Martins Lopes. Análise de assunto: teoria e prática. 2.ed. ver. Brasília: Briquet de Lemos, 2013.

INTERNATIONAL COMMITTEE OF MEDICAL JOURNAL EDITORS. Journal following the ICMJE recommendations. 2017a. Disponível em:

http://www.icmje.org/journals-following-the-icmje-recommendations/journal-listing-requestform/ /. Acesso em: 5 abr. 2019.

INTERNATIONAL COMMITTEE OF MEDICAL JOURNAL EDITORS. Purpose of the recomendations. 2017b Disponível em:

http://www.icmje.org/recommendations/browse/about-the-recommendations/purpose-of-therecommendations.html. Acesso em: 03 fev. 2017.

INTERNATIONAL COMMITTEE OF MEDICAL JOURNAL EDITORS.

Recommendations for the conduct, reporting, editing, and publications of scholarly work in medical journals: updated dec. 2018. Disponível

em:www.icmje.org/recommendations. Acesso em: 5 abr. 2019. 
INTERNATIONAL COMMITTEE OF MEDICAL JOURNAL EDITORS. Samples of formatted references for authors of jornal articles. 2016. Disponível em:

https://www.nlm.nih.gov/bsd/uniform_requirements.html. Acesso em: 03 fev. 2017.

KURAMOTO, H. A informação científica: proposta de um novo modelo para o Brasil. Ci. Inf. Brasília, v.35, n.2, p.91-102, maio/ago. 2006.

LEITE, M. P. F. R. Avaliando a qualidade de revistas científicas para a publicação de resultados de pesquisas e estudos. Rev. Min. Enferm. v.13, n.3. p. 317-219, 2009. Disponível em: http://www.reme.org.br/artigo/detalhes/194. Acesso em: 12 jun. 2016.

MEDICAL SUBJECT HEADINGS. Bethesda (MD): U.S National Library of Medicine: 2019. Disponível em: https://www.nlm.nih.gov/mesh/meshhome.html. Acesso em: 10 jan 2019.

NOVILO ORTIZ, A. Descriptores em ciências de la salud: Decs. Bib. Virt. Cienc Salud, 2010. v.1.

PATRIAS, K.; WENDLING, D. (ed). Citing medicine: the NLM style guide for authors, editors, and publishers. 2.ed. Bethesda (MD): National Library of Medicine (US), 2007. Disponível em: www.nlm.nih.gov/citingmedicine. Acesso em: 12 dez. 2016.

SANTOS, M. R. S.; SAMPAIO, D. B. Normalização na prática: um breve relato sobre normalização e a experiência do grupo de normalizadores. InCID - Rev. Ci. Inf. Doc. v.5, n.1, p.151-165. mar./ago. 2014.

SILVA, A. F. S.; LINS, M. S. V. G. Guia para normalização de trabalhos acadêmicos e científicos produzidos pela Universidade Federal do Amazonas. Manaus: UFAM, 1993.

SILVEIRA, N. C. Os FBRB e a escolha do ponto de acesso pessoal. Persp. Ci. Inf., v.14, n.2, p.108-120, maio./ago.2009.

SIQUEIRA, Jessica Camara. Recursos linguísticos para análise de vocabulário controlado: o caso do SAUSP. Biblionline, v.7, n.2, p.52-62, 2011. Disponível em:

http://www.periodicos.ufpb.br/index.php/biblio/article/view/10211/6938. Acesso em: 8 abr. 2019.

UNIVERSIDADE DO OESTE PAULISTA. Manual de referências: conforme o estilo de Vancouver. Presidente Prudente, SP: UNOESTE, 2011.

VOLPATO, G. L. O método lógico para redação científica. RECIIS - Rev. Eletron.

Comum Inf. Inov. Saúde. v.9, n.1. jan./mar.2015. Disponível em:

http://www.gilsonvolpato.com.br/new/multimidia/artigos/2_6bfbc0fa7d70897e18b1394d48d3

c006.pdf. Acesso em: 23 jun. 2016. 